\title{
GREEN SUPPLIER SELECTION BASED ON CODAS METHOD IN PROBABILISTIC UNCERTAIN LINGUISTIC ENVIRONMENT
}

\author{
Cun $\mathrm{WEI}^{1}$, Jiang WU${ }^{1}$, Yanfeng $\mathrm{GUO}^{2 *}$, Guiwu WEI ${ }^{3}$ \\ ${ }^{1}$ School of Statistics, Southwestern University of Finance and Economics, 611130 Chengdu, P.R. China \\ ${ }^{2}$ School of Finance, Southwestern University of Finance and Economics, 610074 Chengdu, P.R. China \\ ${ }^{3}$ School of Business, Sichuan Normal University, 610101 Chengdu, P.R. China
}

Received 21 February 2020; accepted 15 November 2020

\begin{abstract}
Probabilistic uncertain linguistic sets (PULTSs) have widely been used in MADM or MAGDM. The CODAS method, which is a novel MADM or MAGDM tool, aims to acquire the optimal choice which have the largest Euclidean \& Hamming distances from the NIS. This paper designs the probabilistic uncertain linguistic CODAS (PUL-CODAS) method with sine entropy weight. Finally, a numerical example for green supplier selection is given and the obtained results are compared with some existing models.
\end{abstract}

Keywords: MAGDM, PULTSs, CODAS method, green supplier, selection.

JEL Classification: C43, C61, D81.

\section{Introduction}

The CODAS method was firstly designed by Keshavarz Ghorabaee (2016). It is a novel and useful model used to solve MADM problems with aid of deriving the Euclidean distance and Hamming distances to select the best alternative. Ghorabaee, Amiri, Zavadskas, Hooshmand, and Antuchevičienè (2018) defined the fuzzy CODAS method to select suppliers. Panchal et al. (2017) applied fuzzy CODAS to tackle the maintenance decision issue. (Badi et al., 2018) employed CODAS method to select the optimal desalination plant location in Libya. Yeni and Ozcelik (2019) defined the CODAS method for MAGDM under IVIFSs. Peng and Li (2019) designed the hesitant fuzzy soft CODAS method. Karasan, Bolturk, and Kahraman (2019) proposed neutrosophic CODAS method. Pamucar, Badi, Sanja, and Obradovic (2018) introduced linguistic neutrosophic CODAS method.

Due to certain complexity, experts couldn't depict their preferences through real numbers (Liao \& Xu, 2014a, 2014b, 2014c), thus with help of other mathematical qualitative tool (Beg et al., 2019; Lu \& Wei, 2019; Wang, 2019; Wu et al., 2019a, 2019b). For example, the DMs

${ }^{\star}$ Corresponding author. E-mail: guoyanfeng@swufe.edu.cn 
could employ the linguistic terms to depict satisficing degree of a car (Herrera \& Martinez, 2000b). In order to give qualitative assessment, Herrera and Martinez (2000a) designed the 2TLTSs for calculating along with words. Sohaib, Naderpour, Hussain, and Martinez (2019) defined 2-tuple linguistic TOPSIS for MAGDM issues. Furthermore, Rodriguez, Martinez, and Herrera (2012) designed the HFLTSs which depicts some possible linguistic values. Wei (2019a) defined the GDSM under HFLTSs. Liao, Xu, and Zeng (2015) developed VIKOR model under HFLTSs. Liao, Yang, and Xu (2018b) gave the ELECTRE II model with HFLTS and gave two ELECTRE II model based on the score-deviation and positive and negative ideal.

Furthermore, Pang, Wang, and Xu (2016) defined the PLTSs. Recently, PLTSs have become hot issues for HFLTSs (Liao et al., 2018a; Wei et al., 2018) and HFSs (Xia \& Xu, 2011). Bai, Zhang, Qian, and Wu (2017) formed a comparison model to tackle PLTSs. Gou and Xu (2016) designed some basic operations of PLTSs. Liao, Jiang, $\mathrm{Xu}, \mathrm{Xu}$, and Herrera (2017) defined linear programming model to cope with MADM under PLTSs. Lin, Chen, Liao, and $\mathrm{Xu}$ (2019) defined the ELECTRE II model under PLTSs. Feng, Liu, and Wei (2019) built the PL-QUALIFLEX. Liao, Jiang, Lev, and Fujitac (2019) researched the PL-ELECTRE III model. Bai, Zhang, Shen, Huang, and Fan (2018) built the PLTSs in MAGDM under uncertainty. Jin, Wang, and Xu (2019) gave uncertain PLTSs in GDM. Kobina, Liang, and He (2017) defined the power operators under PLTSs based on power operators (Wei, 2019b; Yager, 2001). Cheng, $\mathrm{Gu}$, and $\mathrm{Xu}$ (2018) studied the GDM under PLTSs setting. Liang, Kobina, and Quan (2018) defined the GRA algorithms for PL-MAGDM under geometric BM (Wang et al., 2018; Wei et al., 2019). Xie, Xu, and Ren (2019) studied the incomplete hybrid probabilistic linguistic problem. $\mathrm{Lu}, \mathrm{Wei}, \mathrm{Wu}$, and Wei (2019) proposed TOPSIS algorithm to solve the PL-MAGDM.

In certain situations, some DMs may depict their preferences through ULTSs (Xu, 2004). Inspired by PLTSs (Pang et al., 2016) and ULTSs (Xu, 2004), Lin, Xu, Zhai, and Yao (2018) defined probabilistic ULTSs (PULTSs). Xie, Ren, Xu, and Wang (2018) depicted some preference relation under PULTSs and designed the distance and similarity. But there are no recent existing literatures to use CODAS method to solve PUL-MAGDM. Therefore, it is very necessary to investigate such issue. The other remaining section of such paper is given. Section 1 reviews the definition of PULTSs. In Section 2, the CODAS method is defined for PUL-MAGDM along with sine entropy weight. In Section 3, a detailed example is developed and some comparative analysis is given. This paper finishes with conclusions in last Section.

\section{Preliminaries}

In such section, some basic mathematical definitions are simply reviewed.

Definition 1 (Gou et al., 2017). Let $L=\left\{l_{\alpha} \mid \alpha=-\theta, \cdots,-2,-1,0,1,2, \cdots \theta\right\}$ be an LTS, the $l_{\alpha}$ could depict the corresponding information with $\beta$ which is defined by using $g$ :

$$
g:\left[l_{-\theta}, l_{\theta}\right] \rightarrow[0,1], \quad g\left(l_{\alpha}\right)=\frac{\alpha+\theta}{2 \theta}=\beta,
$$

$\beta$ could also depicts the equivalent assessing information for $l_{\alpha}$ which is defined with $g^{-1}$ : 


$$
g^{-1}:[0,1] \rightarrow\left[l_{-\theta}, l_{\theta}\right], \quad g^{-1}(\beta)=l_{(2 \beta-1) \theta}=l_{\alpha},
$$

Definition 2 (Pang et al., 2016). Given an LTS $L=\left\{l_{j} \mid j=-\theta, \cdots,-2,-1,0,1,2, \cdots \theta\right\}$, the PLTS is simply defined:

$$
L(p)=\left\{l(\phi)\left(p^{(\phi)}\right) \mid l^{(\phi)} \in L, p^{(\phi)} \geq 0, \phi=1,2, \cdots, \# L(p), \sum_{\phi=1}^{\# L(p)} p^{(\phi)} \leq 1\right\},
$$

where $l^{(\phi)}\left(p^{(\phi)}\right)$ is the $\phi$ th $l^{(\phi)}$ along with corresponding probability values $\left(p^{(\phi)}\right)$, and \#L(p) denotes the number of $L(p)$. The $l^{(\phi)}$ in $L(p)$ are listed with ascending order.

Furthermore, Lin et al. (2018) defined the PULTSs based on ULTSs (Xu, 2004) and PLTSs (Pang et al., 2016).

Definition 3 (Lin et al., 2018). The PULTS is defined:

$$
\operatorname{PULTS}(p)=\left\{\left[L^{\phi}, U^{\phi}\right]\left(p^{\phi}\right) \mid p^{\phi} \geq 0, \phi=1,2, \cdots, \# \operatorname{PULTS}(p), \quad \sum_{\phi=1}^{\# \operatorname{PULTS}(p)} p^{\phi} \leq 1\right\},
$$

where $\left[L^{\phi}, U^{\phi}\right]\left(p^{\phi}\right)$ expresses the uncertain linguistic values $\left[L^{\phi}, U^{\phi}\right]$ with probability values $p^{\phi}, L^{\phi}, U^{\phi}$ are LTSs, $L^{\phi} \leq U^{\phi}$, and \#PULT $(p)$ is the length of PULTS $(p)$.

Definition 4 (Lin et al., 2018). Let $\operatorname{PULTS}_{1}(p)=\left\{\left[L_{1}^{\phi}, U_{1}^{\phi}\right]\left(p_{1}^{\phi}\right) \mid \phi=1,2, \cdots, \# \operatorname{PULTS}_{1}(p)\right\}$ and $\operatorname{PULTS}_{2}(p)=\left\{\left[L_{2}^{\phi}, U_{2}^{\phi}\right]\left(p_{2}^{\phi}\right) \mid \phi=1,2, \cdots, \#\right.$ PULTS $\left._{2}(p)\right\} \quad$ be PULTSs, and the

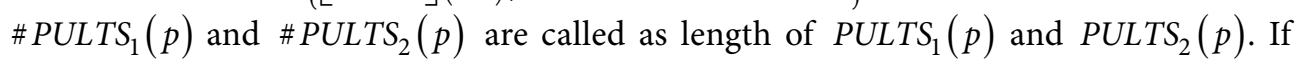
\#PULTS $S_{1}(p)>\# \operatorname{PULTS}_{2}(p)$, then \#PULTS $1(p)-\# P U L T S_{2}(p)$ ULTSs are added to $\operatorname{PULTS}_{2}(p)$. The added ULTSs are the smallest ULTSs in $\operatorname{PULTS}_{2}(p)$ and the corresponding probabilities values of newly added ULTSs are zero.

Definition 5 (Lin et al., 2018). Let $\operatorname{PULTS}(p)=\left\{\left[L^{\phi}, U^{\phi}\right]\left(p^{\phi}\right) \mid \phi=1,2, \cdots, \# \operatorname{PULTS}(p)\right\}$, the defined expected values $\operatorname{EV}(\operatorname{PULTS}(p))$ and corresponding deviation degree $D D(\operatorname{PULTS}(p))$ is proposed:

$$
\begin{gathered}
E V(\operatorname{PULTS}(p))=\frac{\sum_{\phi=1}\left(\frac{g\left(L^{\phi}\right) p^{\phi}+g\left(U^{\phi}\right) p^{\phi}}{2}\right)}{\sum_{\phi=1} p^{\phi}}, \\
D D(\operatorname{PULTSTS}(p))=\frac{\sqrt{\sum_{\phi=1}^{\# \operatorname{PULTS}(p)\left(\frac{g\left(L^{\phi}\right) p^{\phi}+g\left(U^{\phi}\right) p^{\phi}}{2}-E(\operatorname{PULTS}(p))\right)^{2}}}}{\# \sum_{\phi=1}^{\# P U L T S}(p)},
\end{gathered}
$$


Definition 6. Let $\operatorname{PULTS}_{1}(p)=\left\{\left[L_{1}^{\phi}, U_{1}^{\phi}\right]\left(p_{1}^{\phi}\right) \mid \phi=1,2, \cdots, \# \operatorname{PULTS}_{1}(p)\right\}$ and $\operatorname{PULTS}_{2}(p)=$ $\left\{\left[L_{2}^{\phi}, U_{2}^{\phi}\right]\left(p_{2}^{\phi}\right) \mid \phi=1,2, \cdots, \# P U L T S_{2}(p)\right\}$, along with $\# P U L T S_{1}(p)=\# P U L T S_{2}(p)=$ $\# P U L T S(p)$, then the Euclidean distance $E D\left(P U L T S_{1}(p), P U L T S_{2}(p)\right)$ and the Hamming distance $H D\left(\operatorname{PULTS}_{1}(p), \operatorname{PULTS}_{2}(p)\right)$ is listed:

$$
\begin{aligned}
& \operatorname{ED}\left(\operatorname{PULTS}_{1}(p), \operatorname{PULTS}_{2}(p)\right)= \\
& \sqrt{\sum_{\phi=1}^{\# \operatorname{PUTS}(p)}\left(\left|g\left(L_{1}^{\phi}\right) p^{\phi}-g\left(L_{2}^{\phi}\right) p^{\phi}\right|^{2}+\left|g\left(U_{1}^{\phi}\right) p^{\phi}-g\left(U_{2}^{\phi}\right) p^{\phi}\right|^{2}\right) / 2 \# \operatorname{PULTS}(p) ;} \\
& \operatorname{HD}\left(\operatorname{PULTS}(p), \operatorname{PULTS}_{2}(p)\right)= \\
& \frac{\sum_{\phi=1}^{\# \operatorname{PUTS}(p)}\left(\left|g\left(L_{1}^{\phi}\right) p^{\phi}-g\left(L_{2}^{\phi}\right) p^{\phi}\right|+\left|g\left(U_{1}^{\phi}\right) p^{\phi}-g\left(U_{2}^{\phi}\right) p^{\phi}\right|\right)}{2 \# P U L T S(p)} .
\end{aligned}
$$

Then, the sine entropy of PULTS is defined to get unknown attribute weights in MAGDM issue based on the idea of simplified Neutrosophic sine entropy (Cui \& Ye, 2018).

Definition 7. Let $\operatorname{PULTS}(p)=\left\{\operatorname{PULTS}_{1}(p), \operatorname{PULTS}_{2}(p), \cdots, \operatorname{PULTS}_{n}(p)\right\}$ be the PULTS, where $\operatorname{PULTS}_{j}(p)=\left\{\left[L_{j}^{\phi}, U_{j}^{\phi}\right]\left(p_{j}^{\phi}\right) \mid \phi=1,2, \cdots, \# \operatorname{PULTS}_{j}(p)\right\}$ is the $j$-th probabilistic uncertain linguistic number (PULN), $j=1,2, \ldots, n$. Then the probabilistic uncertain linguistic sine entropy measure (PULSEM) is designed:

$$
\begin{aligned}
& \operatorname{PULSEM}(\operatorname{PULTS}(p))= \\
& \frac{1}{2 n \# \operatorname{PULTS}(p)} \sum_{j=1}^{n}\left(\sum_{\phi=1}^{\# \operatorname{PULTS}(p)} \sin \left(g\left(L_{j}^{\phi}\right) p_{j}^{\phi} \pi\right)+\sin \left(g\left(U_{j}^{\phi}\right) p_{j}^{\phi} \pi\right)\right) .
\end{aligned}
$$

\section{CODAS method for PUL-MAGDM issue}

In such part, the PUL-CODAS model for MAGDM is designed. $A=\left\{A_{1}, A_{2}, \cdots, A_{m}\right\}$ is named a group of given alternatives, $G=\left\{G_{1}, G_{2}, \cdots, G_{n}\right\}$ is called a group of given attributes along with weight $w=\left(w_{1}, w_{2}, \cdots, w_{n}\right)$, where $w_{j} \in[0,1], \sum_{j=1}^{n} w_{j}=1$ and $E=\left\{E_{1}, E_{2}, \cdots, E_{q}\right\}$ is named a set of experts. Suppose that $G_{j}$ is evaluated by $E_{k}$ for $A_{i}$ and is expressed as uncertain linguistic variables $\left[L_{i j}^{k}, U_{i j}^{k}\right], i=1,2, \ldots, m, j=1,2, \ldots, n, k=1,2, \ldots, q$.

Then, the CODAS model is devised to deal with PUL-MAGDM issues. The calculating steps are given soon afterwards and the flowchart is given in Figure 1.

Step 1. Convert cost attribute into beneficial attribute. If cost attribute value is $\left[L_{i j}^{k}, U_{i j}^{k}\right]$, then the corresponding beneficial attribute value is $\left[-U_{i j}^{k},-L_{i j}^{k}\right]$. 


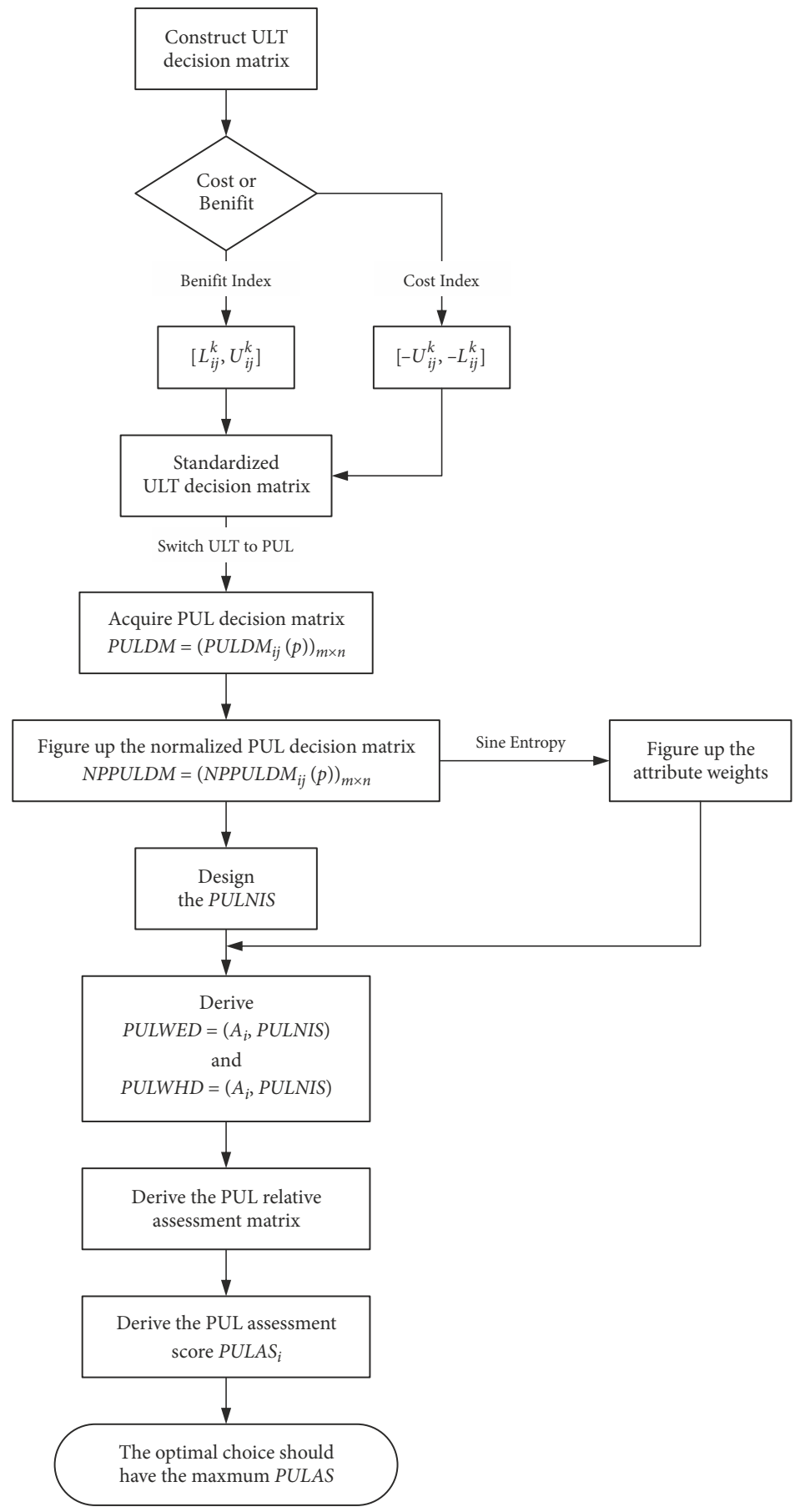

Figure 1. The flowchart of the CODAS method for the PUL-MAGDM 
Step 2. Switch the $\left[L_{i j}^{k}, U_{i j}^{k}\right]$ into PUL matrix $P U L D M=\left(P U L D M_{i j}(p)\right)_{m \times n}, P U L D M_{i j}(p)=$ $\left\{\left[L_{i j}^{\phi}, U_{i j}^{\phi}\right]\left(p_{i j}^{\phi}\right) \mid \phi=1,2, \cdots, \# P U L D M_{i j}(p)\right\}$.

Step 3. Figure up the normalized PUL matrix $N P U L D M=\left(N P U L D M_{i j}(p)\right)_{m \times n}$.

Step 4. Figure up the attributes weight by sine entropy.

Since the uncertainty of one attribute increases, the attribute weight should decrease correspondingly. Thus, we may figure up unknown weights of each attribute based on the sine entropy measure formula Eq. (9). Firstly, the probabilistic uncertain linguistic sine entropy measure (PULSEM) of NPULDM $i j(p)$ are designed as follows:

$$
\begin{aligned}
& \operatorname{PULSEM}\left(\operatorname{NPULDM}_{j}(p)\right)= \\
& \frac{1}{2 m \# N P U L D M_{i j}(p)} \sum_{i=1}^{m}\left(\sum_{\phi=1}^{\# P U L T S}(p) \sin \left(g\left(L_{i j}^{\phi}\right) p_{i j}^{\phi} \pi\right)+\sin \left(g\left(U_{i j}^{\phi}\right) p_{i j}^{\phi} \pi\right)\right)
\end{aligned}
$$

Then, the attribute weights is:

$$
w_{j}=\frac{1-\operatorname{PULSEM}\left(\operatorname{NPULDM}_{j}(p)\right)}{\sum_{j=1}^{n}\left(1-\operatorname{PULSEM}\left(\operatorname{NPULDM}_{j}(p)\right)\right)}, j=1,2, \cdots, n .
$$

Step 5. Design the PULNIS:

$$
\begin{aligned}
& \text { PULNIS }=\left(\text { PULNIS }_{1}, \text { PULNIS }_{2}, \cdots, \text { PULNIS }_{n}\right), \\
& \text { PULNIS }_{j}=\left\{\left[L_{j}^{\phi}, U_{j}^{\phi}\right]\left(p_{j}^{(\phi)}\right) \mid \phi=1,2, \cdots, \# N P U L D M_{i j}(p)\right\}, \\
& E\left(\text { PULNIS }_{j}\right)=\left\{\min _{i} E\left(\text { NPULDM }_{i j}(p)\right)\right\},
\end{aligned}
$$

Step 6. Derive the probabilistic uncertain linguistic weighted Euclidean distance PULWED $\left(A_{i}\right.$, PULNIS $)(i=1,2, \cdots, m)$ and Hamming distance PULWHD $\left(A_{i}\right.$, PULNIS $)$ $(i=1,2, \cdots, m)$ :

$$
\begin{aligned}
& \operatorname{PULWED}\left(A_{i}, \text { PULNIS }\right)=\sum_{j=1}^{n} w_{j} E D\left(N P U L D M_{i j}(p), \text { PULNIS }_{j}\right), \\
& \operatorname{PULWHD}\left(A_{i}, P U L N I S\right)=\sum_{j=1}^{n} w_{j} H D\left(N P U L D M_{i j}(p), \text { PULNIS }_{j}\right), \\
& \text { ED }\left(\operatorname{NPULDM}_{i j}(p), P U L N I S_{j}\right)=
\end{aligned}
$$

$$
\sqrt{\frac{\left(\sum_{\phi=1}^{\# N P U L D M_{i j}(p)}\left(\left|g\left(L_{i j}^{\phi}\right) p_{i j}^{\phi}-g\left(L_{j}^{\phi}\right) p_{j}^{(\phi)}\right|^{2}+\left|g\left(U_{i j}^{\phi}\right) p_{i j}^{\phi}-g\left(U_{j}^{\phi}\right) p_{j}^{(\phi)}\right|^{2}\right)\right)}{2 \# N P U L D M_{i j}(p)}},
$$




$$
\begin{aligned}
& \operatorname{HD}\left(\operatorname{NPULDM}_{i j}(p), \text { PULNIS }_{j}\right)= \\
& \frac{\left(\sum_{\phi=1}^{\# N P U L D M_{i j}(p)}\left(\left|g\left(L_{i j}^{\phi}\right) p_{i j}^{\phi}-g\left(L_{j}^{\phi}\right) p_{j}^{(\phi)}\right|+\left|g\left(U_{i j}^{\phi}\right) p_{i j}^{\phi}-g\left(U_{j}^{\phi}\right) p_{j}^{(\phi)}\right|\right)\right)}{2 \# N P U L D M_{i j}(p)},
\end{aligned}
$$

Step 7. Derive the PUL relative assessment matrix (PULRAM):

$$
\begin{aligned}
& \text { PULRAM }=\left[\text { PULRAM }{ }_{i k}\right]_{m \times m}, \\
& \text { PULRAM }_{i k}=\left(\operatorname{PULWED}\left(A_{i}, \text { PULNIS }\right)-P U L W E D\left(A_{k}, P U L N I S\right)\right)+ \\
& \left(\begin{array}{l}
f\left(\text { PULWED }\left(A_{i}, \text { PULNIS }\right)-P U L W E D\left(A_{k}, \text { PULNIS }\right)\right) \\
\times\left(\text { PULWHD }\left(A_{i}, \text { PULNIS }\right)-P U L W H D\left(A_{k}, \text { PULNIS }\right)\right)
\end{array}\right),
\end{aligned}
$$

where $k \in\{1,2, \ldots, m\}$ and the threshold formula is given by Eq. (21):

$$
f(x)=\left\{\begin{array}{ll}
1 & \text { if }|x| \geq \tau \\
0 & \text { if }|x|<\tau
\end{array},\right.
$$

where the threshold parameter $\tau$ is between 0.01 and 0.05 . In this paper, $\tau=0.02$ are always used to compute (Lin et al., 2018).

Step 8. Derive the PUL assessment score $\operatorname{PULAS}_{i}(i=1,2, \cdots, m)$ by Eq. (22).

$$
\text { PULAS }_{i}=\sum_{k=1}^{m} \text { PULRAM }_{i k} .
$$

Step 9. Sort the alternatives with $P U L A S_{i}$, the optimal choice should have maximum value.

\section{A numerical example and comparative analysis}

\subsection{A numerical example}

In today's world of resource shortage and increasingly serious environmental pollution, facing the strict environmental protection system, how to make the coordinated development of supply chain and environment while pursuing economic benefits will become an important means and decisive factor for enterprises to succeed in market competition (Tavana et al., 2017; Tong, 2017; Wang et al., 2017). Green supply chain management, as a new management mode of core enterprises under sustainable development, has been widely recognized and valued by the academic and business circles (Wei et al., 2020a, 2020b, 2020c, 2020d). Green supplier management is the important part of green supply chain management through the coordination and cooperation with suppliers to achieve cost reduction to reduce resource consumption to improve the environment, and can make the enterprise faster response to market demand, improve the core competitiveness, establish corporate social image. Supplier selection is also an important link along with implementation of green supplier management. Green supplier selection is a very common decision issue (Wang et al., 2020; Wei et al., 2020; 
2020d; Zavadskas et al., 2019; Zhang et al., 2020). Thus, in such section, an example about green supplier selection is given to proof the defined method. There are some green suppliers $G S_{i}(i=1,2,3,4,5)$ for experts to select according to four assessing attributes: (1) $\mathrm{Q}_{1}$ is the environmental competencies; (2) $\mathrm{Q}_{2}$ is transportation cost of suppliers; (3) $\mathrm{Q}_{3}$ is environmental improvement quality; (4) $\mathrm{Q}_{4}$ is financial conditions of suppliers. All these four attributes are adapted from Lei, Wei, Gao, Wu, and Wei (2020). The $\mathrm{Q}_{2}$ is cost index and other indices are beneficial. These potential green suppliers $G S_{i}(i=1,2,3,4,5)$ could be assessed with employing the LTSs

$S=\left\{s_{-3}=\right.$ extremely $\operatorname{poor}(E P), s_{-2}=\operatorname{very} \operatorname{poor}(V P), s_{-1}=\operatorname{poor}(P), s_{0}=\operatorname{medium}(M)$,

$s_{1}=\operatorname{good}(G), s_{2}=\operatorname{very} \operatorname{good}(V G), s_{3}=$ extremely $\left.\operatorname{good}(E G)\right\}$

by five DMs according to these given attributes, as given in the Tables 1-5.

Table 1. The ULTSs through $\mathrm{DM}_{1}$

\begin{tabular}{|c|c|c|c|c|}
\hline Alternatives & $\mathrm{Q}_{1}$ & $\mathrm{Q}_{2}$ & $\mathrm{Q}_{3}$ & $\mathrm{Q}_{4}$ \\
\hline $\mathrm{GS}_{1}$ & {$[\mathrm{M}, \mathrm{G}]$} & {$[\mathrm{M}, \mathrm{G}]$} & {$[\mathrm{G}, \mathrm{VG}]$} & {$[\mathrm{P}, \mathrm{G}]$} \\
\hline $\mathrm{GS}_{2}$ & {$[\mathrm{M}, \mathrm{G}]$} & {$[\mathrm{G}, \mathrm{VG}]$} & {$[\mathrm{M}, \mathrm{G}]$} & {$[\mathrm{VG}, \mathrm{EG}]$} \\
\hline $\mathrm{GS}_{3}$ & {$[\mathrm{VP}, \mathrm{P}]$} & {$[\mathrm{P}, \mathrm{M}]$} & {$[\mathrm{G}, \mathrm{VG}]$} & {$[\mathrm{P}, \mathrm{M}]$} \\
\hline $\mathrm{GS}_{4}$ & {$[\mathrm{G}, \mathrm{VG}]$} & {$[\mathrm{M}, \mathrm{G}]$} & {$[\mathrm{G}, \mathrm{EG}]$} & {$[\mathrm{VG}, \mathrm{EG}]$} \\
\hline $\mathrm{GS}_{5}$ & {$[\mathrm{M}, \mathrm{G}]$} & {$[\mathrm{P}, \mathrm{M}]$} & {$[\mathrm{M}, \mathrm{G}]$} & {$[\mathrm{P}, \mathrm{M}]$} \\
\hline
\end{tabular}

Table 2. The ULTSs through $\mathrm{DM}_{2}$

\begin{tabular}{|c|c|c|c|c|}
\hline Alternatives & $\mathrm{Q}_{1}$ & $\mathrm{Q}_{2}$ & $\mathrm{Q}_{3}$ & $\mathrm{Q}_{4}$ \\
\hline $\mathrm{GS}_{1}$ & {$[\mathrm{M}, \mathrm{G}]$} & {$[\mathrm{G}, \mathrm{VG}]$} & {$[\mathrm{VG}, \mathrm{EG}]$} & {$[\mathrm{P}, \mathrm{G}]$} \\
\hline $\mathrm{GS}_{2}$ & {$[\mathrm{G}, \mathrm{VG}]$} & {$[\mathrm{P}, \mathrm{M}]$} & {$[\mathrm{G}, \mathrm{VG}]$} & {$[\mathrm{G}, \mathrm{VG}]$} \\
\hline $\mathrm{GS}_{3}$ & {$[\mathrm{M}, \mathrm{G}]$} & {$[\mathrm{P}, \mathrm{M}]$} & {$[\mathrm{VG}, \mathrm{EG}]$} & {$[\mathrm{P}, \mathrm{M}]$} \\
\hline $\mathrm{GS}_{4}$ & {$[\mathrm{VG}, \mathrm{EG}]$} & {$[\mathrm{M}, \mathrm{G}]$} & {$[\mathrm{VP}, \mathrm{P}]$} & {$[\mathrm{G}, \mathrm{VG}]$} \\
\hline $\mathrm{GS}_{5}$ & {$[\mathrm{P}, \mathrm{M}]$} & {$[\mathrm{VP}, \mathrm{P}]$} & {$[\mathrm{M}, \mathrm{VG}]$} & {$[\mathrm{M}, \mathrm{VG}]$} \\
\hline
\end{tabular}

Table 3. The ULTSs through $\mathrm{DM}_{3}$

\begin{tabular}{|c|c|c|c|c|}
\hline Alternatives & $\mathrm{Q}_{1}$ & $\mathrm{Q}_{2}$ & $\mathrm{Q}_{3}$ & $\mathrm{Q}_{4}$ \\
\hline $\mathrm{GS}_{1}$ & {$[\mathrm{M}, \mathrm{VG}]$} & {$[\mathrm{M}, \mathrm{G}]$} & {$[\mathrm{VG}, \mathrm{EG}]$} & {$[\mathrm{M}, \mathrm{G}]$} \\
\hline $\mathrm{GS}_{2}$ & {$[\mathrm{M}, \mathrm{G}]$} & {$[\mathrm{G}, \mathrm{VG}]$} & {$[\mathrm{P}, \mathrm{M}]$} & {$[\mathrm{VG}, \mathrm{EG}]$} \\
\hline $\mathrm{GS}_{3}$ & {$[\mathrm{M}, \mathrm{G}]$} & {$[\mathrm{M}, \mathrm{G}]$} & {$[\mathrm{VG}, \mathrm{EG}]$} & {$[\mathrm{G}, \mathrm{VG}]$} \\
\hline $\mathrm{GS}_{4}$ & {$[\mathrm{VG}, \mathrm{EG}]$} & {$[\mathrm{G}, \mathrm{VG}]$} & {$[\mathrm{G}, \mathrm{EG}]$} & {$[\mathrm{VG}, \mathrm{EG}]$} \\
\hline $\mathrm{GS}_{5}$ & {$[\mathrm{G}, \mathrm{VG}]$} & {$[\mathrm{VP}, \mathrm{P}]$} & {$[\mathrm{M}, \mathrm{VG}]$} & {$[\mathrm{M}, \mathrm{VG}]$} \\
\hline
\end{tabular}


Table 4. The ULTSs through $\mathrm{DM}_{4}$

\begin{tabular}{|c|c|c|c|c|}
\hline Alternatives & $\mathrm{Q}_{1}$ & $\mathrm{Q}_{2}$ & $\mathrm{Q}_{3}$ & $\mathrm{Q}_{4}$ \\
\hline $\mathrm{GS}_{1}$ & {$[\mathrm{M}, \mathrm{G}]$} & {$[\mathrm{G}, \mathrm{VG}]$} & {$[\mathrm{VG}, \mathrm{EG}]$} & {$[\mathrm{P}, \mathrm{G}]$} \\
\hline $\mathrm{GS}_{2}$ & {$[\mathrm{G}, \mathrm{VG}]$} & {$[\mathrm{M}, \mathrm{G}]$} & {$[\mathrm{VG}, \mathrm{EG}]$} & {$[\mathrm{VG}, \mathrm{EG}]$} \\
\hline $\mathrm{GS}_{3}$ & {$[\mathrm{VP}, \mathrm{P}]$} & {$[\mathrm{M}, \mathrm{G}]$} & {$[\mathrm{VG}, \mathrm{EG}]$} & {$[\mathrm{P}, \mathrm{M}]$} \\
\hline $\mathrm{GS}_{4}$ & {$[\mathrm{VG}, \mathrm{EG}]$} & {$[\mathrm{M}, \mathrm{G}]$} & {$[\mathrm{G}, \mathrm{EG}]$} & {$[\mathrm{VG}, \mathrm{EG}]$} \\
\hline $\mathrm{GS}_{5}$ & {$[\mathrm{P}, \mathrm{M}]$} & {$[\mathrm{VP}, \mathrm{P}]$} & {$[\mathrm{VG}, \mathrm{EG}]$} & {$[\mathrm{M}, \mathrm{VG}]$} \\
\hline
\end{tabular}

Table 5. The ULTSs through $\mathrm{DM}_{5}$

\begin{tabular}{|c|c|c|c|c|}
\hline Alternatives & $\mathrm{Q}_{1}$ & $\mathrm{Q}_{2}$ & $\mathrm{Q}_{3}$ & $\mathrm{Q}_{4}$ \\
\hline $\mathrm{GS}_{1}$ & {$[\mathrm{M}, \mathrm{G}]$} & {$[\mathrm{VG}, \mathrm{EG}]$} & {$[\mathrm{M}, \mathrm{G}]$} & {$[\mathrm{VP}, \mathrm{P}]$} \\
\hline $\mathrm{GS}_{2}$ & {$[\mathrm{P}, \mathrm{G}]$} & {$[\mathrm{G}, \mathrm{VG}]$} & {$[\mathrm{VG}, \mathrm{EG}]$} & {$[\mathrm{G}, \mathrm{VG}]$} \\
\hline $\mathrm{GS}_{3}$ & {$[\mathrm{G}, \mathrm{VG}]$} & {$[\mathrm{P}, \mathrm{M}]$} & {$[\mathrm{G}, \mathrm{VG}]$} & {$[\mathrm{EP}, \mathrm{VP}]$} \\
\hline $\mathrm{GS}_{4}$ & {$[\mathrm{M}, \mathrm{G}]$} & {$[\mathrm{M}, \mathrm{VG}]$} & {$[\mathrm{G}, \mathrm{VG}]$} & {$[\mathrm{VG}, \mathrm{EG}]$} \\
\hline $\mathrm{GS}_{5}$ & {$[\mathrm{M}, \mathrm{G}]$} & {$[\mathrm{P}, \mathrm{M}]$} & {$[\mathrm{M}, \mathrm{VG}]$} & {$[\mathrm{G}, \mathrm{VG}]$} \\
\hline
\end{tabular}

Then, we employ the PUL-CODAS model designed to choose the optimal green supplier.

Step 1. Convert cost index $\mathrm{Q}_{2}$ into beneficial index (See Tables 6-10). For example, in Table 1 , the ULTS $[M, G]$ is given for alternative $A_{1}$ under $G_{2}$ by the first DM, the converted beneficial attribute value is $[\mathrm{P}, \mathrm{M}]$.

Table 6. The ULTSs through $\mathrm{DM}_{1}$

\begin{tabular}{|c|c|c|c|c|}
\hline Alternatives & $\mathrm{Q}_{1}$ & $\mathrm{Q}_{2}$ & $\mathrm{Q}_{3}$ & $\mathrm{Q}_{4}$ \\
\hline $\mathrm{GS}_{1}$ & {$[\mathrm{M}, \mathrm{G}]$} & {$[\mathrm{P}, \mathrm{M}]$} & {$[\mathrm{G}, \mathrm{VG}]$} & {$[\mathrm{P}, \mathrm{G}]$} \\
\hline $\mathrm{GS}_{2}$ & {$[\mathrm{M}, \mathrm{G}]$} & {$[\mathrm{VP}, \mathrm{P}]$} & {$[\mathrm{M}, \mathrm{G}]$} & {$[\mathrm{VG}, \mathrm{EG}]$} \\
\hline $\mathrm{GS}_{3}$ & {$[\mathrm{VP}, \mathrm{P}]$} & {$[\mathrm{M}, \mathrm{G}]$} & {$[\mathrm{G}, \mathrm{VG}]$} & {$[\mathrm{P}, \mathrm{M}]$} \\
\hline $\mathrm{GS}_{4}$ & {$[\mathrm{G}, \mathrm{VG}]$} & {$[\mathrm{P}, \mathrm{M}]$} & {$[\mathrm{G}, \mathrm{EG}]$} & {$[\mathrm{VG}, \mathrm{EG}]$} \\
\hline $\mathrm{GS}_{5}$ & {$[\mathrm{M}, \mathrm{G}]$} & {$[\mathrm{M}, \mathrm{G}]$} & {$[\mathrm{M}, \mathrm{G}]$} & {$[\mathrm{P}, \mathrm{M}]$} \\
\hline
\end{tabular}

Table 7. The ULTSs through $\mathrm{DM}_{2}$

\begin{tabular}{|c|c|c|c|c|}
\hline Alternatives & $\mathrm{Q}_{1}$ & $\mathrm{Q}_{2}$ & $\mathrm{Q}_{3}$ & $\mathrm{Q}_{4}$ \\
\hline $\mathrm{GS}_{1}$ & {$[\mathrm{M}, \mathrm{G}]$} & {$[\mathrm{VP}, \mathrm{P}]$} & {$[\mathrm{VG}, \mathrm{EG}]$} & {$[\mathrm{P}, \mathrm{G}]$} \\
\hline $\mathrm{GS}_{2}$ & {$[\mathrm{G}, \mathrm{VG}]$} & {$[\mathrm{M}, \mathrm{G}]$} & {$[\mathrm{G}, \mathrm{VG}]$} & {$[\mathrm{G}, \mathrm{VG}]$} \\
\hline $\mathrm{GS}_{3}$ & {$[\mathrm{M}, \mathrm{G}]$} & {$[\mathrm{M}, \mathrm{G}]$} & {$[\mathrm{VG}, \mathrm{EG}]$} & {$[\mathrm{P}, \mathrm{M}]$} \\
\hline $\mathrm{GS}_{4}$ & {$[\mathrm{VG}, \mathrm{EG}]$} & {$[\mathrm{P}, \mathrm{M}]$} & {$[\mathrm{VP}, \mathrm{P}]$} & {$[\mathrm{G}, \mathrm{VG}]$} \\
\hline $\mathrm{GS}_{5}$ & {$[\mathrm{P}, \mathrm{M}]$} & {$[\mathrm{G}, \mathrm{VG}]$} & {$[\mathrm{M}, \mathrm{VG}]$} & {$[\mathrm{M}, \mathrm{VG}]$} \\
\hline
\end{tabular}


Table 8. The ULTSs through $\mathrm{DM}_{3}$

\begin{tabular}{|c|c|c|c|c|}
\hline Alternatives & $\mathrm{Q}_{1}$ & $\mathrm{Q}_{2}$ & $\mathrm{Q}_{3}$ & $\mathrm{Q}_{4}$ \\
\hline $\mathrm{GS}_{1}$ & {$[\mathrm{M}, \mathrm{VG}]$} & {$[\mathrm{P}, \mathrm{M}]$} & {$[\mathrm{VG}, \mathrm{EG}]$} & {$[\mathrm{M}, \mathrm{G}]$} \\
\hline $\mathrm{GS}_{2}$ & {$[\mathrm{M}, \mathrm{G}]$} & {$[\mathrm{VP}, \mathrm{P}]$} & {$[\mathrm{P}, \mathrm{M}]$} & {$[\mathrm{VG}, \mathrm{EG}]$} \\
\hline $\mathrm{GS}_{3}$ & {$[\mathrm{M}, \mathrm{G}]$} & {$[\mathrm{P}, \mathrm{M}]$} & {$[\mathrm{VG}, \mathrm{EG}]$} & {$[\mathrm{G}, \mathrm{VG}]$} \\
\hline $\mathrm{GS}_{4}$ & {$[\mathrm{VG}, \mathrm{EG}]$} & {$[\mathrm{VP}, \mathrm{P}]$} & {$[\mathrm{G}, \mathrm{EG}]$} & {$[\mathrm{VG}, \mathrm{EG}]$} \\
\hline $\mathrm{GS}_{5}$ & {$[\mathrm{G}, \mathrm{VG}]$} & {$[\mathrm{G}, \mathrm{VG}]$} & {$[\mathrm{M}, \mathrm{VG}]$} & {$[\mathrm{M}, \mathrm{VG}]$} \\
\hline
\end{tabular}

Table 9. The ULTSs through $\mathrm{DM}_{4}$

\begin{tabular}{|c|c|c|c|c|}
\hline Alternatives & $\mathrm{Q}_{1}$ & $\mathrm{Q}_{2}$ & $\mathrm{Q}_{3}$ & $\mathrm{Q}_{4}$ \\
\hline $\mathrm{GS}_{1}$ & {$[\mathrm{M}, \mathrm{G}]$} & {$[\mathrm{VP}, \mathrm{P}]$} & {$[\mathrm{VG}, \mathrm{EG}]$} & {$[\mathrm{P}, \mathrm{G}]$} \\
\hline $\mathrm{GS}_{2}$ & {$[\mathrm{G}, \mathrm{VG}]$} & {$[\mathrm{P}, \mathrm{M}]$} & {$[\mathrm{VG}, \mathrm{EG}]$} & {$[\mathrm{VG}, \mathrm{EG}]$} \\
\hline $\mathrm{GS}_{3}$ & {$[\mathrm{VP}, \mathrm{P}]$} & {$[\mathrm{P}, \mathrm{M}]$} & {$[\mathrm{VG}, \mathrm{EG}]$} & {$[\mathrm{P}, \mathrm{M}]$} \\
\hline $\mathrm{GS}_{4}$ & {$[\mathrm{VG}, \mathrm{EG}]$} & {$[\mathrm{P}, \mathrm{M}]$} & {$[\mathrm{G}, \mathrm{EG}]$} & {$[\mathrm{VG}, \mathrm{EG}]$} \\
\hline $\mathrm{GS}_{5}$ & {$[\mathrm{P}, \mathrm{M}]$} & {$[\mathrm{G}, \mathrm{VG}]$} & {$[\mathrm{VG}, \mathrm{EG}]$} & {$[\mathrm{M}, \mathrm{VG}]$} \\
\hline
\end{tabular}

Table 10. The ULTSs through $\mathrm{DM}_{5}$

\begin{tabular}{|c|c|c|c|c|}
\hline Alternatives & $\mathrm{Q}_{1}$ & $\mathrm{Q}_{2}$ & $\mathrm{Q}_{3}$ & $\mathrm{Q}_{4}$ \\
\hline $\mathrm{GS}_{1}$ & {$[\mathrm{M}, \mathrm{G}]$} & {$[\mathrm{EP}, \mathrm{VP}]$} & {$[\mathrm{M}, \mathrm{G}]$} & {$[\mathrm{VP}, \mathrm{P}]$} \\
\hline $\mathrm{GS}_{2}$ & {$[\mathrm{P}, \mathrm{G}]$} & {$[\mathrm{VP}, \mathrm{P}]$} & {$[\mathrm{VG}, \mathrm{EG}]$} & {$[\mathrm{G}, \mathrm{VG}]$} \\
\hline $\mathrm{GS}_{3}$ & {$[\mathrm{G}, \mathrm{VG}]$} & {$[\mathrm{M}, \mathrm{G}]$} & {$[\mathrm{G}, \mathrm{VG}]$} & {$[\mathrm{EP}, \mathrm{VP}]$} \\
\hline $\mathrm{GS}_{4}$ & {$[\mathrm{M}, \mathrm{G}]$} & {$[\mathrm{VP}, \mathrm{M}]$} & {$[\mathrm{G}, \mathrm{VG}]$} & {$[\mathrm{VG}, \mathrm{EG}]$} \\
\hline $\mathrm{GS}_{5}$ & {$[\mathrm{M}, \mathrm{G}]$} & {$[\mathrm{M}, \mathrm{G}]$} & {$[\mathrm{M}, \mathrm{VG}]$} & {$[\mathrm{G}, \mathrm{VG}]$} \\
\hline
\end{tabular}

Step 2. Convert the ULTSs into PULTSs (See Table 11).

Table 11. Decision matrix with PULTSs

\begin{tabular}{|c|c|c|}
\hline Alternatives & $\mathrm{Q}_{1}$ & $\mathrm{Q}_{2}$ \\
\hline $\mathrm{GS}_{1}$ & $\left\{\left\langle\left[l_{0}, l_{1}\right] 0.8\right\rangle,\left\langle\left[l_{0}, l_{2}\right] 0.2\right\rangle\right\}$ & $\left\{\begin{array}{l}\left\langle\left[l_{0}, l_{1}\right] 0.4\right\rangle,\left\langle\left[l_{1}, l_{2}\right] 0.4\right\rangle, \\
\left\langle\left[l_{2}, l_{3}\right] 0.2\right\rangle\end{array}\right\}$ \\
\hline $\mathrm{GS}_{2}$ & $\left\{\begin{array}{l}\left\langle\left[l_{-1}, l_{1}\right] 0.2\right\rangle,\left\langle\left[l_{0}, l_{1}\right] 0.4\right\rangle, \\
\left\langle\left[l_{1}, l_{2}\right] 0.4\right\rangle\end{array}\right\}$ & $\left\{\begin{array}{l}\left\langle\left[l_{-1}, l_{0}\right] 0.2\right\rangle,\left\langle\left[l_{0}, l_{1}\right] 0.2\right\rangle, \\
\left\langle\left[l_{1}, l_{2}\right] 0.6\right\rangle\end{array}\right\}$ \\
\hline $\mathrm{GS}_{3}$ & $\left\{\begin{array}{l}\left\langle\left[l_{-2}, l_{-1}\right] 0.4\right\rangle,\left\langle\left[l_{0}, l_{1}\right] 0.4\right\rangle, \\
\left\langle\left[l_{1}, l_{2}\right] 0.2\right\rangle\end{array}\right\}$ & $\left\{\left\langle\left[l_{-1}, l_{0}\right] 0.6\right\rangle,\left\langle\left[l_{0}, l_{1}\right] 0.4\right\rangle\right\}$ \\
\hline $\mathrm{GS}_{4}$ & $\left\{\begin{array}{l}\left\langle\left[l_{0}, l_{1}\right] 0.2\right\rangle,\left\langle\left[l_{1}, l_{2}\right] 0.2\right\rangle, \\
\left\langle\left[l_{2}, l_{3}\right] 0.6\right\rangle\end{array}\right\}$ & $\left\{\begin{array}{l}\left\langle\left[l_{0}, l_{1}\right] 0.6\right\rangle,\left\langle\left[l_{1}, l_{2}\right] 0.2\right\rangle, \\
\left\langle\left[l_{0}, l_{2}\right] 0.2\right\rangle\end{array}\right\}$ \\
\hline $\mathrm{GS}_{5}$ & $\left\{\begin{array}{l}\left\langle\left[l_{-1}, l_{0}\right] 0.4\right\rangle,\left\langle\left[l_{0}, l_{1}\right] 0.4\right\rangle, \\
\left\langle\left[l_{1}, l_{2}\right] 0.2\right\rangle\end{array}\right\}$ & $\left\{\left\langle\left[l_{-2}, l_{-1}\right] 0.6\right\rangle,\left\langle\left[l_{-1}, l_{0}\right] 0.4\right\rangle\right\}$ \\
\hline
\end{tabular}


End of Table 11

\begin{tabular}{|c|c|c|}
\hline Alternatives & $\mathrm{Q}_{3}$ & $\mathrm{Q}_{4}$ \\
\hline $\mathrm{GS}_{1}$ & $\left\{\begin{array}{l}\left\langle\left[l_{0}, l_{1}\right] 0.2\right\rangle,\left\langle\left[l_{1}, l_{2}\right] 0.2\right\rangle, \\
\left\langle\left[l_{2}, l_{3}\right] 0.6\right\rangle\end{array}\right\}$ & $\left\{\begin{array}{l}\left\langle\left[l_{-2}, l_{-1}\right] 0.2\right\rangle,\left\langle\left[l_{0}, l_{1}\right] 0.2\right\rangle, \\
\left\langle\left[l_{-1}, l_{1}\right] 0.6\right\rangle\end{array}\right\}$ \\
\hline $\mathrm{GS}_{2}$ & $\left\{\begin{array}{l}\left\{\left[l_{-1}, l_{0}\right] 0.2\right\rangle,\left\langle\left[l_{0}, l_{1}\right] 0.2\right\rangle, \\
\left\langle\left[l_{2}, l_{3}\right] 0.6\right\rangle\end{array}\right\}$ & $\left\{\left\langle\left[l_{1}, l_{2}\right] 0.4\right\rangle,\left\langle\left[l_{2}, l_{3}\right] 0.6\right\rangle\right\}$ \\
\hline $\mathrm{GS}_{3}$ & $\left\{\left\langle\left[l_{1}, l_{2}\right] 0.4\right\rangle,\left\langle\left[l_{2}, l_{3}\right] 0.6\right)\right\}$ & $\left\{\begin{array}{l}\left\langle\left[l_{-3}, l_{-2}\right] 0.2\right\rangle,\left\langle\left[l_{-1}, l_{0}\right] 0.6\right\rangle, \\
\left\langle\left[l_{1}, l_{2}\right] 0.2\right\rangle\end{array}\right.$ \\
\hline $\mathrm{GS}_{4}$ & $\left\{\begin{array}{l}\left\langle\left[l_{-2}, l_{-1}\right] 0.2\right\rangle,\left\langle\left[l_{1}, l_{2}\right] 0.2\right\rangle, \\
\left\langle\left[l_{1}, l_{3}\right] 0.6\right\rangle\end{array}\right\}$ & $\left\{\begin{array}{l}\left\langle\left[l_{1}, l_{2}\right] 0.0\right\rangle,\left\langle\left[l_{1}, l_{2}\right] 0.2\right\rangle, \\
\left\langle\left[l_{2}, l_{3}\right] 0.8\right\rangle\end{array}\right\}$ \\
\hline $\mathrm{GS}_{5}$ & $\left\{\begin{array}{l}\left\langle\left[l_{0}, l_{2}\right] 0.6\right\rangle,\left\langle\left[l_{0}, l_{1}\right] 0.2\right\rangle, \\
\left\langle\left[l_{2}, l_{3}\right] 0.2\right\rangle\end{array}\right\}$ & $\left\{\begin{array}{l}\left\langle\left[l_{-1}, l_{0}\right] 0.2\right\rangle,\left\langle\left[l_{1}, l_{2}\right] 0.2\right\rangle, \\
\left\langle\left[l_{0}, l_{2}\right] 0.6\right\rangle\end{array}\right\}$ \\
\hline
\end{tabular}

Step 3. Compute the normalized PULTSs (Table 12).

Table 12. Normalized PULTSs

\begin{tabular}{|c|c|c|}
\hline Alternatives & $\mathrm{Q}_{1}$ & $\mathrm{Q}_{2}$ \\
\hline $\mathrm{GS}_{1}$ & $\left\{\begin{array}{l}\left\langle\left[l_{0}, l_{1}\right] 0\right\rangle,\left\langle\left[l_{0}, l_{1}\right] 0.9\right\rangle, \\
\left\langle\left[l_{1}, l_{2}\right] 0.1\right\rangle\end{array}\right\}$ & $\left\{\begin{array}{l}\left\{\left[l_{0}, l_{1}\right] 0.4\right\rangle,\left\langle\left[l_{1}, l_{2}\right] 0.4\right\rangle, \\
\left\langle\left[l_{2}, l_{3}\right] 0.2\right\rangle\end{array}\right\}$ \\
\hline $\mathrm{GS}_{2}$ & $\left\{\begin{array}{l}\left\langle\left[l_{-1}, l_{0}\right] 0.1\right\rangle,\left\langle\left[l_{0}, l_{1}\right] 0.5\right\rangle, \\
\left\langle\left[l_{1}, l_{2}\right] 0.4\right\rangle\end{array}\right\}$ & $\left\{\begin{array}{l}\left\langle\left[l_{-1}, l_{0}\right] 0.2\right\rangle,\left\langle\left[l_{0}, l_{1}\right] 0.2\right\rangle, \\
\left\langle\left[l_{1}, l_{2}\right] 0.6\right\rangle\end{array}\right\}$ \\
\hline $\mathrm{GS}_{3}$ & $\left\{\begin{array}{l}\left\langle\left[l_{-2}, l_{-1}\right] 0.4\right\rangle,\left\langle\left[l_{0}, l_{1}\right] 0.4\right\rangle, \\
\left\langle\left[l_{1}, l_{2}\right] 0.2\right\rangle\end{array}\right.$ & $\left\{\begin{array}{l}\left\{\left[l_{-1}, l_{0}\right] 0\right\rangle,\left\langle\left[l_{-1}, l_{0}\right] 0.6\right\rangle, \\
\left\langle\left[l_{0}, l_{1}\right] 0.4\right\rangle\end{array}\right\}$ \\
\hline $\mathrm{GS}_{4}$ & $\left\{\begin{array}{l}\left\langle\left[l_{0}, l_{1}\right] 0.2\right\rangle,\left\langle\left[l_{1}, l_{2}\right] 0.2\right\rangle, \\
\left\langle\left[l_{2}, l_{3}\right] 0.6\right\rangle\end{array}\right\}$ & $\left\{\begin{array}{l}\left\langle\left[l_{0}, l_{1}\right] 0\right\rangle,\left\langle\left[l_{0}, l_{1}\right] 0.7\right\rangle, \\
\left\langle\left[l_{1}, l_{2}\right] 0.3\right\rangle\end{array}\right\}$ \\
\hline $\mathrm{GS}_{5}$ & $\left\{\begin{array}{l}\left\langle\left[l_{-1}, l_{0}\right] 0.4\right\rangle,\left\langle\left[l_{0}, l_{1}\right] 0.4\right\rangle, \\
\left\langle\left[l_{1}, l_{2}\right] 0.2\right\rangle\end{array}\right\}$ & $\left\{\begin{array}{l}\left\langle\left[l_{-2}, l_{-1}\right] 0.6\right\rangle,\left\langle\left[l_{-2}, l_{-1}\right] 0.6\right\rangle, \\
\left\langle\left[l_{-1}, l_{0}\right] 0.4\right\rangle\end{array}\right.$ \\
\hline
\end{tabular}


End of Table 12

\begin{tabular}{|c|c|c|}
\hline Alternatives & $\mathrm{Q}_{3}$ & $\mathrm{Q}_{4}$ \\
\hline $\mathrm{GS}_{1}$ & $\left\{\begin{array}{l}\left\langle\left[l_{0}, l_{1}\right] 0.2\right\rangle,\left\langle\left[l_{1}, l_{2}\right] 0.2\right\rangle, \\
\left\langle\left[l_{2}, l_{3}\right] 0.6\right\rangle\end{array}\right\}$ & $\left\{\begin{array}{l}\left\langle\left[l_{-2}, l_{-1}\right] 0.2\right\rangle,\left\langle\left[l_{-1}, l_{0}\right] 0.3\right\rangle, \\
\left\langle\left[l_{0}, l_{1}\right] 0.5\right\rangle\end{array}\right.$ \\
\hline $\mathrm{GS}_{2}$ & $\left\{\begin{array}{l}\left\langle\left[l_{-1}, l_{0}\right] 0.2\right\rangle,\left\langle\left[l_{0}, l_{1}\right] 0.2\right\rangle, \\
\left\langle\left[l_{2}, l_{3}\right] 0.6\right\rangle\end{array}\right\}$ & $\left\{\begin{array}{l}\left\langle\left[l_{1}, l_{2}\right] 0\right\rangle,\left\langle\left[l_{1}, l_{2}\right] 0.4\right\rangle, \\
\left\langle\left[l_{2}, l_{3}\right] 0.6\right\rangle\end{array}\right\}$ \\
\hline $\mathrm{GS}_{3}$ & $\left\{\begin{array}{l}\left\langle\left[l_{1}, l_{2}\right] 0\right\rangle,\left\langle\left[l_{1}, l_{2}\right] 0.4\right\rangle, \\
\left\langle\left[l_{2}, l_{3}\right] 0.6\right\rangle\end{array}\right\}$ & $\left\{\begin{array}{l}\left\langle\left[l_{-3}, l_{-2}\right] 0.2\right\rangle,\left\langle\left[l_{-1}, l_{0}\right] 0.6\right\rangle, \\
\left\langle\left[l_{1}, l_{2}\right] 0.2\right\rangle\end{array}\right.$ \\
\hline $\mathrm{GS}_{4}$ & $\left\{\begin{array}{l}\left\langle\left[\left[l_{-2}, l_{-1}\right] 0.2\right\rangle,\left\langle\left[l_{1}, l_{2}\right] 0.5\right\rangle,\right. \\
\left\langle\left[l_{2}, l_{3}\right] 0.3\right\rangle\end{array}\right.$ & $\left\{\begin{array}{l}\left\langle\left[l_{1}, l_{2}\right] 0.0\right\rangle,\left\langle\left[l_{1}, l_{2}\right] 0.2\right\rangle, \\
\left\langle\left[l_{2}, l_{3}\right] 0.8\right\rangle\end{array}\right\}$ \\
\hline $\mathrm{GS}_{5}$ & $\left\{\begin{array}{l}\left\{\left[l_{0}, l_{1}\right] 0.5\right\rangle,\left\langle\left[l_{1}, l_{2}\right] 0.3\right\rangle, \\
\left\langle\left[l_{2}, l_{3}\right] 0.2\right\rangle\end{array}\right\}$ & $\left\{\begin{array}{l}\left\{\left[l_{-1}, l_{0}\right] 0.2\right\rangle,\left\langle\left[l_{0}, l_{1}\right] 0.3\right\rangle, \\
\left\langle\left[l_{1}, l_{2}\right] 0.5\right\rangle\end{array}\right\}$ \\
\hline
\end{tabular}

Step 4. Derive the attributes weight from Eq. (10)-(11), the attributes weight is given in Table 13.

Table 13. The attributes weight

\begin{tabular}{|c|c|c|c|c|}
\hline Weight & $w_{1}$ & $w_{2}$ & $w_{3}$ & $w_{4}$ \\
\hline$w$ & 0.2535 & 0.2621 & 0.2110 & 0.2734 \\
\hline
\end{tabular}

Step 5. Obtain the PULNIS (Table 14).

Table 14. PULNIS

\begin{tabular}{|c|c|c|}
\hline & $\mathrm{Q}_{1}$ & $\mathrm{Q}_{2}$ \\
\hline PULNIS & $\left\{\begin{array}{l}\left\langle\left[l_{-2}, l_{-1}\right] 0.4\right\rangle,\left\langle\left[l_{0}, l_{1}\right] 0.4\right\rangle, \\
\left\langle\left[l_{1}, l_{2}\right] 0.2\right\rangle\end{array}\right.$ & $\left\{\begin{array}{l}\left.\left\langle\left\langle l_{-2}, l_{-1}\right] 0.0\right\rangle,\left\langle\left[l_{-2}, l_{-1}\right] 0.6\right\rangle,\right\} \\
\left\langle\left[l_{-1}, l_{0}\right] 0.4\right\rangle\end{array}\right.$
\end{tabular}

$\left.\begin{array}{|c|c|c|}\hline & \mathrm{Q}_{3} & \mathrm{Q}_{4} \\ \hline \text { PULNIS } & \left\{\begin{array}{l}\left\langle\left[l_{-1}, l_{0}\right] 0.2\right\rangle,\left\langle\left[l_{0}, l_{1}\right] 0.6\right\rangle, \\ \left\langle\left[l_{1}, l_{2}\right] 0.2\right\rangle\end{array}\right. & \left\{\begin{array}{l}\left\langle\left[l_{-3}, l_{-2}\right] 0.2\right\rangle,\left\langle\left[l_{-1}, l_{0}\right] 0.6\right\rangle, \\ \left\langle\left[l_{1}, l_{2}\right] 0.2\right\rangle\end{array}\right.\end{array}\right\}$


Step 6. Compute the PULWED(GS, PULNIS $)$ and PULWHD $\left(G S_{i}, P U L N I S\right)(i=1,2,3,4,5)$ through Eqs. (15)-(18) (See Table 15).

Table 15. PULWED $\left(G S_{i}\right.$, PULNIS $)$ and PULWHD $\left(A_{i}\right.$, PULNIS $)$

\begin{tabular}{|c|c|c|}
\hline Alternatives & PULWED $\left(\right.$ GS $_{i}$, PULNIS $)$ & PULWHD(GS, PULNIS $)$ \\
\hline $\mathrm{GS}_{1}$ & 0.1828 & 0.1463 \\
\hline $\mathrm{GS}_{2}$ & 0.2016 & 0.1446 \\
\hline $\mathrm{GS}_{3}$ & 0.1153 & 0.0521 \\
\hline $\mathrm{GS}_{4}$ & 0.2323 & 0.1466 \\
\hline $\mathrm{GS}_{5}$ & 0.1017 & 0.0648 \\
\hline
\end{tabular}

Step 7. Compute the PULRAM $=\left[P U L R A M_{i k}\right]_{5 \times 5}$ through Eqs. (19)-(21) (Table 16).

Table 16. PULRAM $=\left[P U L R A M_{i k}\right]_{5 \times 5}$

\begin{tabular}{|c|c|c|c|c|c|}
\hline & $\mathrm{GS}_{1}$ & $\mathrm{GS}_{2}$ & $\mathrm{GS}_{3}$ & $\mathrm{GS}_{4}$ & $\mathrm{GS}_{5}$ \\
\hline $\mathrm{GS}_{1}$ & 0.0000 & -0.0188 & 0.1617 & -0.0498 & 0.1626 \\
\hline $\mathrm{GS}_{2}$ & 0.0188 & 0.0000 & 0.1788 & -0.0327 & 0.1797 \\
\hline $\mathrm{GS}_{3}$ & -0.1617 & -0.1788 & 0.0000 & -0.2115 & 0.0136 \\
\hline $\mathrm{GS}_{4}$ & 0.0498 & 0.0327 & 0.2115 & 0.0000 & 0.2123 \\
\hline $\mathrm{GS}_{5}$ & -0.1626 & -0.1797 & -0.0136 & -0.2123 & 0.0000 \\
\hline
\end{tabular}

Step 8. Calculate the $\operatorname{PULAS}_{i}(i=1,2,3,4,5)$ by Eq. (22) (See Table 17).

Table 17. PULAS decision matrix

\begin{tabular}{|c|c|c|c|c|c|}
\hline Alternatives & $\mathrm{GS}_{1}$ & $\mathrm{GS}_{2}$ & $\mathrm{GS}_{3}$ & $\mathrm{GS}_{4}$ & $\mathrm{GS}_{5}$ \\
\hline PULAS & 0.2556 & 0.3446 & -0.5383 & 0.5062 & -0.5682 \\
\hline
\end{tabular}

Step 9. According to $\operatorname{PULAS}_{i}(i=1,2,3,4,5)$, the order is $G S_{4}>G S_{2}>G S_{1}>G S_{3}>G S_{5}$. That's to say, $G S_{4}$ is the optimal alternative.

\subsection{Comparative analysis}

Then, the PUL-CODAS is compared with ULWA operator (Xu, 2004), PUL-TOPSIS (Lin et al., 2018) and PULWA (Lin et al., 2018).

\subsubsection{Compared with ULWA}

Firstly, we deal with such example by using the ULWA (Xu, 2004) with same weight to aggregate these ULTSs into a group matrix (See Table 18). 
Table 18. Group uncertain linguistic matrix

\begin{tabular}{|c|c|c|c|c|}
\hline Alternatives & $\mathrm{Q}_{1}$ & $\mathrm{Q}_{2}$ & $\mathrm{Q}_{3}$ & $\mathrm{Q}_{4}$ \\
\hline $\mathrm{GS}_{1}$ & {$\left[l_{0.0}, l_{1.2}\right]$} & {$\left[l_{0.8}, l_{1.8}\right]$} & {$\left[l_{1.4}, l_{2.4}\right]$} & {$\left[l_{-1}, l_{0.6}\right]$} \\
\hline $\mathrm{GS}_{2}$ & {$\left[l_{0.2}, l_{1.4}\right]$} & {$\left[l_{0.4}, l_{1.4}\right]$} & {$\left[l_{1}, l_{2}\right]$} & {$\left[l_{1.6}, l_{2.4}\right]$} \\
\hline $\mathrm{GS}_{3}$ & {$\left[l_{-0.6}, l_{0.4}\right]$} & {$\left[l_{-0.6}, l_{0.4}\right]$} & {$\left[l_{1.6}, l_{2.4}\right]$} & {$\left[l_{-1}, l_{0}\right]$} \\
\hline $\mathrm{GS}_{4}$ & {$\left[l_{1.4}, l_{2.4}\right]$} & {$\left[l_{0.2}, l_{1.4}\right]$} & {$\left[l_{0.4}, l_{2}\right]$} & {$\left[l_{1.8}, l_{2.8}\right]$} \\
\hline $\mathrm{GS}_{5}$ & {$\left[l_{-0.2}, l_{0.8}\right]$} & {$\left[l_{-1.6}, l_{-0.6}\right]$} & {$\left[l_{0.4}, l_{2}\right]$} & {$\left[l_{0}, l_{1.6}\right]$} \\
\hline
\end{tabular}

The weight is: $w_{1}=0.2535, w_{2}=0.2621, w_{3}=0.2110, w_{4}=0.2734$, then the total value is derived by employing ULWA (Xu, 2004).

$$
\begin{aligned}
& Z_{1}(w)=\left[s_{0.2318}, s_{1.4465}\right], Z_{2}(w)=\left[s_{0.8040}, s_{1.800}\right], Z_{3}(w)=\left[s_{-0.2451}, s_{0.7127}\right], \\
& Z_{4}(w)=\left[s_{0.9838}, s_{2.1628}\right], Z_{5}(w)=\left[s_{-0.3857}, s_{0.9049}\right] .
\end{aligned}
$$

Then, the score of five potential alternative are derived through Definition 9 (Lin et al., 2018):

$$
\begin{aligned}
& E\left(Z_{1}(w)\right)=s_{0.8391}, E\left(Z_{2}(w)\right)=s_{1.3020}, E\left(Z_{3}(w)\right)=s_{0.2338} \\
& E\left(Z_{4}(w)\right)=s_{1.5733}, E\left(Z_{5}(w)\right)=s_{0.2596} .
\end{aligned}
$$

Furthermore, the order is derived: $G S_{4}>G S_{2}>G S_{1}>G S_{5}>G S_{3}$. Thus, we could obtain the optimal green supplier $G S_{4}$.

\subsubsection{Compared with PUL-TOPSIS}

Then, the PUL-CODAS is compared with the PUL-TOPSIS model (Lin et al., 2018), then the derived result is obtained (Table 19). Thus, the best green supplier is $G S_{4}$.

Table 19. The calculating results through PUL-TOPSIS

\begin{tabular}{|l|l|}
\hline \multicolumn{1}{|c|}{ TOPSIS } & \multicolumn{1}{c|}{ Calculating results } \\
\hline $\begin{array}{l}\text { The distances } \\
\text { from PULPIS }\end{array}$ & $d_{1}^{+}=0.5423, d_{2}^{+}=0.4089, d_{3}^{+}=0.6594, d_{4}^{+}=0.1651, d_{5}^{+}=0.7746$ \\
\hline $\begin{array}{l}\text { The distances } \\
\text { from PULNIS }\end{array}$ & $d_{1}^{-}=0.6250, d_{2}^{-}=0.7830, d_{3}^{-}=0.4272, d_{4}^{-}=0.8823, d_{5}^{-}=0.4599$ \\
\hline Closeness coefficients & $C I_{1}=-2.5773, C I_{2}=-1.5899, C I_{3}=-3.5110, C I_{4}=0.0000, C I_{5}=-4.1714$ \\
\hline Ordering & $G S_{4}>G S_{2}>G S_{1}>G S_{3}>G S_{5}$ \\
\hline
\end{tabular}

\subsubsection{Compared with PULWA}

Finally, the PUL-CODAS is compared with PULWA (Lin et al., 2018), the attributes weight is: $w_{1}=0.2535, w_{2}=0.2621, w_{3}=0.2110, w_{4}=0.2734$, then the total value is derived through PULWA operator. 


$$
\begin{aligned}
& Z_{1}(w)=\left\{\left[s_{-0.1094}, s_{0.0924}\right],\left[s_{0.0650}, s_{0.5222}\right],\left[s_{0.3834}, s_{0.7245}\right]\right\}, \\
& Z_{2}(w)=\left\{\left[s_{-0.1200}, s_{0.0000}\right],\left[s_{0.1094}, s_{0.4401}\right],\left[s_{0.8399}, s_{1.3893}\right]\right\}, \\
& Z_{3}(w)=\left\{\left[s_{-0.3668}, s_{-0.2107}\right],\left[s_{-0.2369}, s_{0.2702}\right],\left[s_{0.3586}, s_{0.6954}\right]\right\}, \\
& Z_{4}(w)=\left\{\left[s_{-0.0844}, s_{0.0085}\right],\left[s_{0.2109}, s_{0.6053}\right],\left[s_{0.9468}, s_{1.4595}\right]\right\}, \\
& Z_{5}(w)=\left\{\left[s_{-0.1561}, s_{0.1055}\right],\left[s_{-0.2513}, s_{0.1527}\right],\left[s_{0.1669}, s_{0.5014}\right]\right\} .
\end{aligned}
$$

Then, the score of these five alternatives are derived through Definition 9 (Lin, et al., 2018):

$$
\begin{aligned}
& E\left(Z_{1}(w)\right)=s_{0.1865}, E\left(Z_{2}(w)\right)=s_{0.2954}, E\left(Z_{3}(w)\right)=s_{0.0566}, \\
& E\left(Z_{4}(w)\right)=s_{0.3496}, E\left(Z_{5}(w)\right)=s_{0.0577} .
\end{aligned}
$$

The order is: $G S_{4}>G S_{2}>G S_{1}>G S_{5}>G S_{3}$ and the optimal green supplier is $G S_{4}$.

\section{Conclusions}

In such paper, we developed the CODAS model for MAGDM based on PULTSs and sine entropy weight. Firstly, the Euclidean and Hamming distance under PULTSs are introduced. Then, the CODAS method is proposed for PUL-MAGDM and its main merit is that it highlights Euclidean and Hamming distance from PULNIS. Finally, an example analysis about green supplier selection is utilized to show the defined algorithms and some detailed comparative analysis are used to elucidate the effectiveness in practical decision making.

However, there still remains some unfinished work to be done. Since the computational process of the PULTSs is complicated, we need to further investigate the operations of PULTSs. Except that, the consensus analysis between different groups should be take into account. In future, we are also going to carry out researches on these two aspects and devote to apply the designed methods to other fields, such as pattern recognition, industrial engineering, E-commerce, and so on. At the same time, the corresponding application of the designed algorithms under PULTSs are studied through some other uncertain MADM and uncertain settings and the basic concept of PULTSs could be employed to expand some other fuzzy settings with help of their corresponding probability.

\section{References}

Badi, I., Ballem, M., \& Shetwan, A. (2018). Site selection of desalination plant in Libya by using Combinative Distance-Based Assessment (CODAS) method. International Journal for Quality Research, 12(3), 609-624.

Bai, C. Z., Zhang, R., Qian, L. X., \& Wu, Y. N. (2017). Comparisons of probabilistic linguistic term sets for multi-criteria decision making. Knowledge-Based Systems, 119, 284-291. https://doi.org/10.1016/j.knosys.2016.12.020

Bai, C. Z., Zhang, R., Shen, S., Huang, C. F., \& Fan, X. (2018). Interval-valued probabilistic linguistic term sets in multi-criteria group decision making. International Journal of Intelligent Systems, 33(6), 1301-1321. https://doi.org/10.1002/int.21983 
Beg, I., Jamil, R. N., \& Rashid, T. (2019). Diminishing Choquet hesitant 2-tuple linguistic aggregation operator for multiple attributes group decision making. International Journal of Analysis and Applications, 17(1), 76-104.

Cheng, X., Gu, J., \& Xu, Z. S. (2018). Venture capital group decision-making with interaction under probabilistic linguistic environment. Knowledge-Based Systems, 140, 82-91. https://doi.org/10.1016/j.knosys.2017.10.030

Cui, W. H., \& Ye, J. ( 2018). Improved symmetry measures of simplified neutrosophic sets and their decision-making method based on a sine entropy weight model. Symmetry-Basel, 10(6), 225. https://doi.org/10.3390/sym10060225

Feng, X. Q., Liu, Q., \& Wei, C. P. (2019). Probabilistic linguistic QUALIFLEX approach with possibility degree comparison. Journal of Intelligent \& Fuzzy Systems, 36(1), 719-730. https://doi.org/10.3233/JIFS-172112

Ghorabaee, M. K., Amiri, M., Zavadskas, E. K., Hooshmand, R., \& Antuchevičienè, J. (2018). Fuzzy extension of the CODAS method for multi-criteria market segment evaluation. Journal of Business Economics \& Management, 18(1), 1-19. https://doi.org/10.3846/16111699.2016.1278559

Gou, X. J., \& Xu, Z. S. (2016). Novel basic operational laws for linguistic terms, hesitant fuzzy linguistic term sets and probabilistic linguistic term sets. Information Sciences, 372, 407-427. https://doi.org/10.1016/j.ins.2016.08.034

Gou, X. J., Xu, Z. S., \& Liao, H. C. (2017). Multiple criteria decision making based on Bonferroni means with hesitant fuzzy linguistic information. Soft Computing, 21, 6515-6529.

https://doi.org/10.1007/s00500-016-2211-1

Herrera, F., \& Martinez, L. (2000a). A 2-tuple fuzzy linguistic representation model for computing with words. IEEE Transactions on Fuzzy Systems, 8(6), 746-752.

https://doi.org/10.1109/91.890332

Herrera, F., \& Martinez, L. (2000b). An approach for combining linguistic and numerical information based on the 2-tuple fuzzy linguistic representation model in decision-making. International Journal of Uncertainty Fuzziness and Knowledge-Based Systems, 8(5), 539-562. https://doi.org/10.1142/S0218488500000381

Jin, C., Wang, H., \& Xu, Z. S. (2019). Uncertain probabilistic linguistic term sets in group decision making. International Journal of Fuzzy Systems, 21, 1241-1258. https://doi.org/10.1007/s40815-019-00619-9

Karasan, A., Bolturk, E., \& Kahraman, C. (2019). A novel neutrosophic CODAS method: Selection among wind energy plant locations. Journal of Intelligent \& Fuzzy Systems, 36(2), 1491-1504. https://doi.org/10.3233/JIFS-181255

Keshavarz Ghorabaee, M., Zavadskas, E. K., Turskis, Z., \& Antucheviciene, J. (2016). A new combinative distance-based assessment (CODAS) method for multi-criteria decision-making. Economic Computation \& Economic Cybernetics Studies \& Research, 50(3), 25-44.

Kobina, A., Liang, D. C., \& He, X. (2017). Probabilistic linguistic power aggregation operators for multicriteria group decision making. Symmetry-Basel, 9(12), 320. https://doi.org/10.3390/sym9120320

Lei, F., Wei, G. W., Gao, H., Wu, J., \& Wei, C. (2020). TOPSIS method for developing supplier selection with probabilistic linguistic information. International Journal of Fuzzy Systems, 22, 749-759. https://doi.org/10.1007/s40815-019-00797-6

Liang, D. C., Kobina, A., \& Quan, W. (2018). Grey relational analysis method for probabilistic linguistic multi-criteria group decision-making based on geometric Bonferroni mean. International Journal of Fuzzy Systems, 20, 2234-2244. https://doi.org/10.1007/s40815-017-0374-2

Liao, H. C., Jiang, L. S., Lev, B., \& Fujitac, H. (2019). Novel operations of PLTSs based on the disparity degrees of linguistic terms and their use in designing the probabilistic linguistic ELECTRE III method. Applied Soft Computing, 80, 450-464. https://doi.org/10.1016/j.asoc.2019.04.018 
Liao, H. C., Jiang, L. S., Xu, Z. H., Xu, J. P., \& Herrera, F. (2017). A linear programming method for multiple criteria decision making with probabilistic linguistic information. Information Sciences, 415, 341-355. https://doi.org/10.1016/j.ins.2017.06.035

Liao, H. C., Si, G. S., Xu, Z. S., \& Fujita, H. (2018a). Hesitant fuzzy linguistic preference utility set and its application in selection of fire rescue plans. International Journal of Environmental Research and Public Health, 15(4), 664. https://doi.org/10.3390/ijerph15040664

Liao, H. C., \& Xu, Z. S. (2014a). Multi-criteria decision making with intuitionistic fuzzy PROMETHEE. Journal of Intelligent \& Fuzzy Systems, 27(4), 1703-1717. https://doi.org/10.3233/IFS-141137

Liao, H. C., \& Xu, Z. S. (2014b). Satisfaction degree based interactive decision making under hesitant fuzzy environment with incomplete weights. International Journal of Uncertainty Fuzziness and Knowledge-Based Systems, 22(4), 553-572. https://doi.org/10.1142/S0218488514500275

Liao, H. C., \& Xu, Z. S. (2014c). Subtraction and division operations over hesitant fuzzy sets. Journal of Intelligent \& Fuzzy Systems, 27(1), 65-72. https://doi.org/10.3233/IFS-130978

Liao, H. C., Xu, Z. S., \& Zeng, X. J. (2015). Hesitant fuzzy linguistic VIKOR method and its application in qualitative multiple criteria decision making. IEEE Transactions on Fuzzy Systems, 23(5), 1343-1355. https://doi.org/10.1109/TFUZZ.2014.2360556

Liao, H. C. C., Yang, L. Y. Y., \& Xu, Z. S. S. (2018b). Two new approaches based on ELECTRE II to solve the multiple criteria decision making problems with hesitant fuzzy linguistic term sets. Applied Soft Computing, 63, 223-234. https://doi.org/10.1016/j.asoc.2017.11.049

Lin, M. W., Chen, Z. Y., Liao, H. C., \& Xu, Z. S. (2019). ELECTRE II method to deal with probabilistic linguistic term sets and its application to edge computing. Nonlinear Dynamics, 96, 2125-2143. https://doi.org/10.1007/s11071-019-04910-0

Lin, M. W., Xu, Z. S., Zhai, Y. L., \& Yao, Z. Q. (2018). Multi-attribute group decision-making under probabilistic uncertain linguistic environment. Journal of the Operational Research Society, 69(2), 157-170. https://doi.org/10.1057/s41274-017-0182-y

Lu, J. P., \& Wei, C. (2019). TODIM method for performance appraisal on social-integration-based rural reconstruction with interval-valued intuitionistic fuzzy information. Journal of Intelligent \& Fuzzy Systems, 37(2), 1731-1740. https://doi.org/10.3233/JIFS-179236

Lu, J. P., Wei, C., Wu, J., \& Wei, G. W. (2019). TOPSIS method for probabilistic linguistic MAGDM with entropy weight and its application to supplier selection of new agricultural machinery products. Entropy, 21(10), 953. https://doi.org/10.3390/e21100953

Pamucar, D., Badi, I., Sanja, K., \& Obradovic, R. (2018). A novel approach for the selection of powergeneration technology using a linguistic neutrosophic CODAS method: A case study in Libya. Energies, 11(9), 2489. https://doi.org/10.3390/en11092489

Panchal, D., Chatterjee, P., Shukla, R. K., Choudhury, T., \& Tamosaitiene, J. (2017). Integrated FUZZY AHP-CODAS framework for maintenance decision in urea fertilizer industry. Economic Computation and Economic Cybernetics Studies and Research, 51, 179-196.

Pang, Q., Wang, H., \& Xu, Z. S. (2016). Probabilistic linguistic term sets in multi-attribute group decision making. Information Sciences, 369, 128-143. https://doi.org/10.1016/j.ins.2016.06.021

Peng, X. D., \& Li, W. Q. (2019). Algorithms for hesitant fuzzy soft decision making based on revised aggregation operators, WDBA and CODAS. Journal of Intelligent \& Fuzzy Systems, 36(6), 6307-6323. https://doi.org/10.3233/JIFS-182594

Rodriguez, R. M., Martinez, L., \& Herrera, F. (2012). Hesitant fuzzy linguistic term sets for decision making. IEEE Transactions on Fuzzy Systems, 20(1), 109-119. https://doi.org/10.1109/TFUZZ.2011.2170076

Sohaib, O., Naderpour, M., Hussain, W., \& Martinez, L. (2019). Cloud computing model selection for e-commerce enterprises using a new 2-tuple fuzzy linguistic decision-making method. Computers \& Industrial Engineering, 132, 47-58. https://doi.org/10.1016/j.cie.2019.04.020 
Tavana, M., Yazdani, M., \& Di Caprio, D. (2017). An application of an integrated ANP-QFD framework for sustainable supplier selection. International Journal of Logistics-Research and Applications, 20(3), 254-275. https://doi.org/10.1080/13675567.2016.1219702

Tong, Y. (2017). Model for evaluating the green supply chain performance under low-carbon agricultural economy environment with 2-tuple linguistic information. Journal of Intelligent \& Fuzzy Systems, 32(3), 2717-2723. https://doi.org/10.3233/JIFS-16802

Wang, J., Wei, G. W., Wei, C., \& Wei, Y. (2020). MABAC method for multiple attribute group decision making under q-rung orthopair fuzzy environment. Defence Technology, 16(1), 208-216. https://doi.org/10.1016/j.dt.2019.06.019

Wang, J., Wei, G. W., \& Wei, Y. (2018). Models for green supplier selection with some 2-tuple linguistic neutrosophic number Bonferroni mean operators. Symmetry-Basel, 10(5), 131. https://doi.org/10.3390/sym10050131

Wang, K. Q., Liu, H. C., Liu, L. P., \& Huang, J. (2017). Green supplier evaluation and selection using cloud model theory and the QUALIFLEX method. Sustainability, 9(5), 688. https://doi.org/10.3390/su9050688

Wang, R. (2019). Research on the application of the financial investment risk appraisal models with some interval number muirhead mean operators. Journal of Intelligent \& Fuzzy Systems, 37(2), 1741-1752. https://doi.org/10.3233/JIFS-179237

Wei, C. P., Rodriguez, R. M., \& Martinez, L. (2018). Uncertainty measures of extended hesitant fuzzy linguistic term sets. IEEE Transactions on Fuzzy Systems, 26(3), 1763-1768. https://doi.org/10.1109/TFUZZ.2017.2724023

Wei, G., Lei, F., Lin, R., Wang, R., Wei, Y., Wu, J., \& Wei, C. (2020). Algorithms for probabilistic uncertain linguistic multiple attribute group decision making based on the GRA and CRITIC method: Application to location planning of electric vehicle charging stations. Economic Research-Ekonomska Istrazivanja, 33(1), 828-846. https://doi.org/10.1080/1331677X.2020.1734851

Wei, G. W. (2019a). The generalized dice similarity measures for multiple attribute decision making with hesitant fuzzy linguistic information. Economic Research-Ekonomska Istrazivanja, 32(1), 1498-1520. https://doi.org/10.1080/1331677X.2019.1637765

Wei, G. W. (2019b). Pythagorean fuzzy Hamacher Power aggregation operators in multiple attribute decision making. Fundamenta Informaticae, 166(1), 57-85. https://doi.org/10.3233/FI-2019-1794

Wei, G. W., He, Y., Lei, F., Wu, J., \& Wei, C. (2020a). MABAC method for multiple attribute group decision making with probabilistic uncertain linguistic information. Journal of Intelligent \& Fuzzy Systems, 39(3), 3315-3327. https://doi.org/10.3233/JIFS-191688

Wei, G. W., He, Y., Lei, F., Wu, J., Wei, C., \& Guo, Y. F. (2020b). Green supplier selection with an uncertain probabilistic linguistic MABAC method. Journal of Intelligent \& Fuzzy Systems, 39(3), 3125-3136. https://doi.org/10.3233/JIFS-191584

Wei, G. W., Lu, J. P., Wei, C., \& Wu, J. (2020c). Probabilistic linguistic GRA method for multiple attribute group decision making. Journal of Intelligent \& Fuzzy Systems, 38(4), 4721-4732. https://doi.org/10.3233/JIFS-191416

Wei, G. W., Wang, J., Lu, J. P., Wu, J., Wei, C., Alsaadi, F. E., \& Hayat, T. (2020d). VIKOR method for multiple criteria group decision making under 2-tuple linguistic neutrosophic environment. Economic Research-Ekonomska Istrazivanja, 33(1), 3185-3208. https://doi.org/10.1080/1331677X.2019.1691036

Wei, G. W., Wang, R., Wang, J., Wei, C., \& Zhang, Y. (2019). Methods for evaluating the technological innovation capability for the high-tech enterprises with generalized interval neutrosophic number Bonferroni mean operators. IEEE Access, 7, 86473-86492.

https://doi.org/10.1109/ACCESS.2019.2925702 
Wu, L. P., Gao, H., \& Wei, C. (2019a). VIKOR method for financing risk assessment of rural tourism projects under interval-valued intuitionistic fuzzy environment. Journal of Intelligent \& Fuzzy Systems, 37(2), 2001-2008. https://doi.org/10.3233/JIFS-179262

Wu, L. P., Wang, J., \& Gao, H. (2019b). Models for competiveness evaluation of tourist destination with some interval-valued intuitionistic fuzzy Hamy mean operators. Journal of Intelligent and Fuzzy Systems, 36(6), 5693-5709. https://doi.org/10.3233/JIFS-181545

Xia, M. M., \& Xu, Z. S. (2011). Hesitant fuzzy information aggregation in decision making. International Journal of Approximate Reasoning, 52(3), 395-407. https://doi.org/10.1016/j.ijar.2010.09.002

Xie, W. Y., Ren, Z. L., Xu, Z. S., \& Wang, H. (2018). The consensus of probabilistic uncertain linguistic preference relations and the application on the virtual reality industry. Knowledge-Based Systems, 162, 14-28. https://doi.org/10.1016/j.knosys.2018.07.016

Xie, W. Y., Xu, Z. S., \& Ren, Z. L. (2019). An analysis on the influence of Chinese "New Four Inventions" under the incomplete hybrid probabilistic linguistic environment. International Journal of Fuzzy Systems, 21, 1349-1366. https://doi.org/10.1007/s40815-019-00635-9

$\mathrm{Xu}, \mathrm{Z}$. S. (2004). Uncertain linguistic aggregation operators based approach to multiple attribute group decision making under uncertain linguistic environment. Information Sciences, 168(1-4), 171-184. https://doi.org/10.1016/j.ins.2004.02.003

Yager, R. R. (2001). The power average operator. IEEE Transactions on Systems, Man, and Cybernetics Part A, 31(6), 724-731. https://doi.org/10.1109/3468.983429

Yeni, F. B., \& Ozcelik, G. (2019). Interval-valued Atanassov intuitionistic fuzzy CODAS method for multi criteria group decision making problems. Group Decision and Negotiation, 28, 433-452. https://doi.org/10.1007/s10726-018-9603-9

Zavadskas, E. K., Antucheviciene, J., \& Chatterjee, P. (2019). Multiple-Criteria Decision-Making $(\mathrm{MCDM})$ techniques for business processes information management. Information, 10(1), 4. https://doi.org/10.3390/info10010004

Zhang, S., Wei, G., Alsaadi, F. E., Hayat, T., Wei, C., \& Zhang, Z. (2020). MABAC method for multiple attribute group decision making under picture 2-tuple linguistic environment. Soft Computing, 24, 5819-5829. https://doi.org/10.1007/s00500-019-04364-x 


\section{APPENDIX}

Abbreviations

CODAS - Combinative distance-based assessment;

DMs - Decision makers;

MAGDM - Multiple attribute group decision making;

NIS - Negative-ideal solution;

PUL - Probabilistic uncertain linguistic;

PULTSs - Probabilistic uncertain linguistic sets;

ULTSs - Uncertain linguistic terms sets;

PUL-MAGDM - Probabilistic uncertain linguistic MAGDM;

PULNIS - Probabilistic uncertain linguistic negative ideal solution;

IVIFSs - Interval-valued intuitionistic fuzzy sets;

2TLTSs - 2-tuple linguistic term sets;

PL-MAGDM - Probabilistic linguistic MAGDM;

GDSM - - Generalized dice similarity measures;

HFLTSs - Hesitant fuzzy linguistic term sets. 